\title{
Anderson-Kondo Lattice Hamiltonian from the Anderson-Lattice Model: A Modified Schrieffer-Wolff Transformation and the Effective Exchange Interactions
}

\begin{abstract}
E. KĄDZIELAWA-MAJOR* AND J. SPAŁEK
Marian Smoluchowski Institute of Physics, Jagiellonian University, W.S. Reymonta 4, 30-059 Kraków, Poland

We derive the Anderson-Kondo lattice model by applying canonical perturbation expansion for the Andersonlattice model in direct space. The transformation is carried out up to the fourth order by a modified Schrieffer-Wolff transformation: we separate the part of hybridization term responsible for the high-energy processes (involving the largest in-the-system intraatomic Coulomb interaction between $f$ electrons) and replace it with the virtual processes in higher orders. The higher-order processes lead to three separate exchange interactions. The obtained Hamiltonian contains both the Kondo $(f-c)$ and the superexchange $(f-f)$ interactions, as well as a residual hybridization responsible for the heavy-quasiparticle formation. This effective Hamiltonian can be used to analyze the magnetic or the paired states, as well their coexistence in heavy-fermion systems. The magnitudes of both the Kondo exchange and the superexchange integrals are estimated as a function of bare hybridization magnitude.
\end{abstract}

DOI: 10.12693/APhysPolA.126.A-100

PACS: 71.10.Fd, 71.20.Eh, 71.27.+a, 71.70.Gm

\section{Introduction}

In this paper we present our main results concerning the canonical perturbation expansion for the Andersonlattice model in direct space, by transforming out only a part of the $f-c$ hybridization term and replacing it with the virtual processes in higher orders, which in turn yield the effective $f-c, f-f$, and $c-c$ interactions. The calculations are carried out up to the fourth order, taking into account both two- and three-site processes. These results elaborate and correct the earlier results [1]. We also estimate the magnitude of the derived exchange integrals. The present results provide an effective model for subsequent consideration of magnetism and real-space pairing in heavy-fermion systems $[2,3]$. The results represent an application of the modified Schrieffer-Wolff transformation, that leads, among others, to the itineracy of originally localized $f$ electrons.

\section{Model}

The basic feature of Anderson-lattice model is the hybridization term $V_{i m}$ representing the quantummechanical mixing between the two types of electrons: the atomic $(f)$ and the conduction $(c)$ states. We assume that $\left|V_{i m}\right| \ll U$, where $U$ is the magnitude of the $f$ - $f$ Coulomb interaction in the same atomic $f$-state. Other Coulomb interactions (in the conduction band and between bands) are disregarded. Additionally, we put

* corresponding author; e-mail: ewa.kadzielawa@uj.edu.pl $\epsilon_{f} \sim V_{i m}$, which means that the atomic level is located below, but not too far from the Fermi surface. Therefore, one can calculate nontrivial corrections in small parameter $V_{i m} / U$ to the electronic $f$ and $c$ states if the strong Coulomb interaction $\sim U$ and the hybridization $\sim V_{i m}$ are included.

The starting Anderson-lattice Hamiltonian in the site (real-space) language reads

$$
\begin{aligned}
\mathcal{H} & =\sum_{\substack{m n \sigma \\
m \neq n}}\left(t_{m n}-\mu \delta_{m n}\right) \hat{c}_{m \sigma}^{\dagger} \hat{c}_{n \sigma}+\epsilon_{f} \sum_{i \sigma} \hat{N}_{i \sigma} \\
& +U \sum_{i} \hat{N}_{i \uparrow} \hat{N}_{i \downarrow}+\sum_{i m \sigma}\left(V_{i m} \hat{f}_{i \sigma}^{\dagger} \hat{c}_{m \sigma}+V_{i m}^{*} \hat{c}_{m \sigma}^{\dagger} \hat{f}_{i \sigma}\right),
\end{aligned}
$$

where $\hat{c}_{m \sigma}^{\dagger}, \hat{c}_{m \sigma}$ are creation and annihilation operators of electrons in $c$-state in real-space representation ( $m$ is the site number and $\sigma$ the spin), $\hat{f}_{i \sigma}^{\dagger}, \hat{f}_{i \sigma}$ are creation and annihilation operators of $f$-electrons on $i$-th site with spin $\sigma, \hat{N}_{i \sigma} \equiv \hat{f}_{i \sigma}^{\dagger} \hat{f}_{i \sigma}$ is the number of $f$-electrons on site $i, t_{m n}$ is hopping integral for $c$-electrons, $\epsilon_{f}$ is the bare energy of the originally localized $4 f$ electrons, $V_{i m}$ is hybridization matrix element and $U$ is intraatomic Coulomb interaction (the high-energy scale in the system).

The starting point in the derivation of the effective Hamiltonian via a canonical perturbation expansion (introduced for Anderson-lattice model in [1]) is a division of the hybridization term into two parts. Namely, we divide the term into two, reflecting the low- and the high-energy processes, i.e., those which do not and do involve energy $U$, respectively, as depicted schematically in Fig. 1. In formal language, it amounts to separating the hybridization term in the following manner: 


$$
\hat{f}_{i \sigma}^{\dagger} \hat{c}_{m \sigma} \equiv\left(1-\hat{N}_{i \bar{\sigma}}\right) \hat{f}_{i \sigma}^{\dagger} \hat{c}_{m \sigma}+\hat{N}_{i \bar{\sigma}} \hat{f}_{i \sigma}^{\dagger} \hat{c}_{m \sigma}
$$

Next, by treating as a perturbation only the part connected with high-energy processes, i.e., $\sim\left(\hat{N}_{i \bar{\sigma}} \hat{f}_{i \sigma}^{\dagger} \hat{c}_{m \sigma}+\right.$ H.c.), we calculate explicitly the effective Hamiltonian using the canonical perturbation expansion up to the fourth order. The low-energy part remains unchanged and represents a residual hybridization, which will introduce, among others, the itineracy of the starting (bare) localized $f$ states. In general, the canonical perturbation expansion method allows for differentiation between the two terms in $(1)$, which are of the same order $\left(\sim V_{i m}\right)$. The differentiation constitutes the main difference between the present transformation and that introduced originally by Schrieffer and Wolff [4]. It will lead to far reaching consequences, e.g., the itineracy of originally atomic $(f)$ electrons.

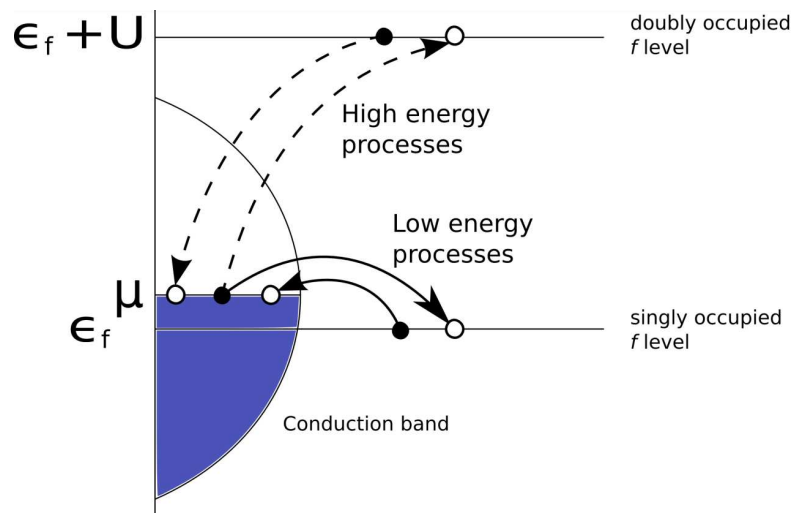

Fig. 1. Low- and high-energy interband hopping processes in direct space induced by the hybridization between $f$ and $c$ states. Only the high-energy $f-c$ mixing processes (involving energy $U$ ) are transformed out and replaced by exchange processes in the second and the fourth orders. Low-energy processes remain unchanged in effective Hamiltonian as residual hybridization. In effect, such transformation differs from the standard Schrieffer-Wolff transformation, where both terms are transformed out.

It should be noted that in the present approach the number of $f$ electrons $\sum_{i} N_{i}$ is not conserved, neither before nor after the transformation. Instead, only the total number of electrons in the system, $n_{i}^{(\mathrm{e})}=N_{i}+n_{i}$ is fixed, where $n_{i} \equiv \sum_{\sigma} c_{i \sigma}^{\dagger} c_{i \sigma}$. This last circumstance allows for an itineracy of strongly correlated $f$ electrons; it allows to represent one of the principal differences with the Schrieffer-Wolff approach.

\section{Canonical perturbation expansion: a brief summary}

To develop the canonical perturbation expansion (CPE) we proceed as follows [1]. Due to the fact that important are the double occupancies of $f$ electrons on the same site, we project them out from Hamiltonian with the help of operators $P_{l}$ :

$$
\sum_{l} P_{l}=1 \quad \text { and } \quad P_{l} P_{l^{\prime}}=\delta_{l l^{\prime}} P_{l} .
$$

Operators $P_{l}$ project the states onto subspace with $(l-1)$ double occupancies in the system of $f$ sites. We redefine initial Anderson-lattice model using projection operators $P_{l}$ in the following manner:

$$
\begin{aligned}
& \mathcal{H}_{0} \equiv P_{1} \mathcal{H} P_{1}+P_{2} \mathcal{H} P_{2}, \\
& \mathcal{H}_{1} \equiv P_{1} \mathcal{H} P_{2}+P_{2} \mathcal{H} P_{1} .
\end{aligned}
$$

In this representation, $\mathcal{H}_{1}$ describes the processes changing by one number of double occupancies

$$
P_{2} \mathcal{H} P_{1}=\left(P_{1} \mathcal{H} P_{2}\right)^{\dagger} \equiv \sum_{i m \sigma} V_{i m} \hat{N}_{i \bar{\sigma}} \hat{f}_{i \sigma}^{\dagger} \hat{c}_{m \sigma} .
$$

In reality, only the effective Hamiltonian projected onto $P_{1}$ subspace will matter; the role of the higher-energy subspaces will show up through virtual processes only.

Now, we introduce the canonical transformation of (1) using the transformation generator $\mathcal{S}$ of the form

$$
\tilde{\mathcal{H}}(\varepsilon)=\mathrm{e}^{-\mathrm{i} \varepsilon \mathcal{S}}\left(\mathcal{H}_{0}+\varepsilon \mathcal{H}_{1}\right) \mathrm{e}^{+\mathrm{i} \varepsilon \mathcal{S}},
$$

where $\varepsilon$ is a parameter, which groups the terms of the same order of expansion in $V_{i m}$ (at the end we put $\varepsilon=1$ ). Expanding the exponential functions into a Taylor series and eliminating the linear term $\sim \varepsilon$ by setting the physical condition

$$
\mathcal{H}_{1}=\mathrm{i}\left[\mathcal{S}, \mathcal{H}_{0}\right]
$$

we obtain up to the fourth order

$$
\begin{aligned}
& \tilde{\mathcal{H}}(\varepsilon)=\mathcal{H}_{0}-\frac{\mathrm{i}}{2} \varepsilon^{2}\left[\mathcal{S}, \mathcal{H}_{1}\right]-\frac{1}{3} \varepsilon^{3}\left[\mathcal{S},\left[\mathcal{S}, \mathcal{H}_{1}\right]\right] \\
& +\frac{\mathrm{i}}{8} \varepsilon^{4}\left[\mathcal{S},\left[\mathcal{S},\left[\mathcal{S}, \mathcal{H}_{1}\right]\right]\right]+\mathcal{O}\left(\varepsilon^{5}\right) .
\end{aligned}
$$

With the use of the definition of projection operators we can find form of $P_{l} \mathcal{S} P_{l+1}$ from condition (8), by putting $P_{l} \mathcal{S}^{(0)} P_{l+1}=0$ and iterating the solution [1]. Finally, we obtain

$$
\begin{aligned}
& P_{l} \mathcal{S}^{(n \rightarrow \infty)} P_{l+1}= \\
& \quad-\mathrm{i}\left(P_{l} \mathcal{H}_{1} P_{l+1}\right)\left(P_{l+1} \mathcal{H}_{0} P_{l+1}-P_{l} \mathcal{H}_{0} P_{l}\right)^{-1} .
\end{aligned}
$$

Let us note that $P_{l} \mathcal{S} P_{l} \sim P_{l}$, thus we can always choose $\mathcal{S}$ in such a way that $P_{l} \mathcal{S} P_{l}=0$, because if we project (8) with operator $P_{l}$ on both sides we obtain that $P_{l} \mathcal{S} P_{l}$ commutes with $\mathcal{H}_{0}$.

In the atomic limit, the difference $P_{l+1} \mathcal{H}_{0} P_{l+1}-$ $P_{l} \mathcal{H}_{0} P_{l}$ can be replaced by mean value of energy difference between subspaces with $l$ and $(l-1)$ double occupancies. By making this approximation, we neglect renormalization of the low-energy hybridization processes by the higher order contributions (i.e., neglect the terms $\sim V_{i m}$ in the denominator of (10)). In effect, we have

$$
\begin{aligned}
& P_{l+1} \mathcal{H}_{0} P_{l+1}-P_{l} \mathcal{H}_{0} P_{l} \approx\left\langle P_{l+1} \mathcal{H}_{0} P_{l+1}\right\rangle-\left\langle P_{l} \mathcal{H}_{0} P_{l}\right\rangle= \\
& U+\epsilon_{f}-\mu \equiv U+\epsilon_{f} .
\end{aligned}
$$

Finally, by projecting out the expansion introduced by expression (9) on the subspace without double occupan- 
cies, the effective Hamiltonian can be obtained in the form

$$
\begin{aligned}
P_{1} \tilde{\mathcal{H}} P_{1} \approx P_{1} \mathcal{H}_{0} P_{1}-\frac{1}{U+\epsilon_{f}} P_{1} \mathcal{H}_{1} P_{2} \mathcal{H}_{1} P_{1} \\
+\frac{1}{\left(U+\epsilon_{f}\right)^{3}}\left(P_{1} \mathcal{H}_{1} P_{2} \mathcal{H}_{1} P_{1} \mathcal{H}_{1} P_{2} \mathcal{H}_{1} P_{1}\right. \\
\left.-\frac{1}{2} P_{1} \mathcal{H}_{1} P_{2} \mathcal{H}_{1} P_{3} \mathcal{H}_{1} P_{2} \mathcal{H}_{1} P_{1}\right),
\end{aligned}
$$

where we have put $\varepsilon=1$. Let us note that the third-order term is always zero, because we have chosen that $P_{l} \mathcal{S} P_{l}=0$.

The term $P_{1} \mathcal{H}_{1} P_{2} \mathcal{H}_{1} P_{1}$ describes virtual process in the second order in which in intermediate state a single double occupancy occurs. In the fourth order two different types of processes appear: those with passing through the subspace $\left(P_{1}\right)$ without double occupancies and those with passing through that subspace with up to two double occupancies $\left(P_{3}\right)$.

In what follows we restrict ourselves to the most interesting part, that is to the Hamiltonian projected onto the subspace without double occupancies (12). This part will be discussed in detail, because it is helpful in determining the ground state for different magnetic and superconducting phases of heavy fermions with nominal $4 f^{1}$ starting configuration ( $\mathrm{Ce}^{3+}$ ions).

\section{Results: Kondo $(f-c)$ and superexchange $(f-f)$ integrals}

An explicit form of the effective Hamiltonian can be found, if we carry out a careful analysis of all possible processes, which can show up in the second and the fourth orders of the expansion. After collecting the all possible diagrams containing two- and three-site processes (examples are shown in Fig. 2), we evaluate them

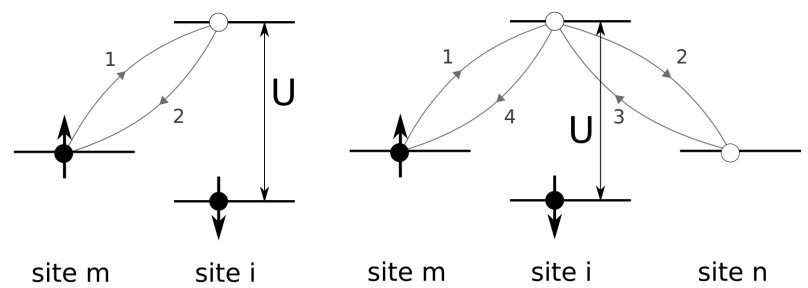

Fig. 2. Examples of processes in the second (left) and the fourth (right) orders of the CPE expansion.

using definitions (4)-(6). In effect, the complete effective Hamiltonian (12) with projected out double occupancies $\left(\hat{\mathcal{H}}_{\text {eff }} \simeq P_{1} \tilde{\mathcal{H}} P_{1}\right)$ has the following form:

$$
\begin{aligned}
& \hat{\mathcal{H}}_{\mathrm{eff}} \simeq \sum_{m \neq n, \sigma}\left(t_{m n}-\mu \delta_{m n}\right) \hat{c}_{m \sigma}^{\dagger} \hat{c}_{n \sigma}+\epsilon_{f} \sum_{i, \sigma} \hat{\nu}_{i \sigma} \\
& \quad+\sum_{i, m, \sigma}\left(V_{i m}\left(1-\hat{N}_{i \bar{\sigma}}\right) \hat{f}_{i \sigma}^{\dagger} \hat{c}_{m \sigma}+\text { H.c. }\right) \\
& \quad+\sum_{i, m} J_{i m}^{(\mathrm{K})}\left(\hat{\boldsymbol{S}}_{i} \cdot \hat{\boldsymbol{s}}_{m}-\frac{\hat{n}_{m} \hat{\nu}_{i}}{4}\right)
\end{aligned}
$$

$$
\begin{aligned}
& +\sum_{i \neq j, \sigma} J_{i j}^{(\mathrm{H})}\left(\hat{\boldsymbol{S}}_{i} \cdot \hat{\boldsymbol{S}}_{j}-\frac{\hat{\nu}_{i} \hat{\nu}_{j}}{4}\right) \\
& +2 \mathrm{i} \sum_{\langle m i\rangle\langle m j\rangle} J_{i j}^{(\mathrm{H})}\left(1+\frac{n_{f}}{n_{\mathrm{c}}}\right) \hat{\boldsymbol{s}}_{m} \cdot\left(\hat{\boldsymbol{S}}_{j} \times \hat{\boldsymbol{S}}_{i}\right),
\end{aligned}
$$

where the projected particle-number operators are $\nu_{i \sigma} \equiv$ $\left(1-\hat{N}_{i \bar{\sigma}}\right) \hat{N}_{i \sigma}$, and $\nu_{i} \equiv \sum_{\sigma} \nu_{i \sigma} ; \hat{\boldsymbol{S}}_{i}$ and $\hat{\boldsymbol{s}}_{m}$ are the local spin operators in the fermion representation for $f$ and $c$ electrons, respectively; $n_{\mathrm{c}} \equiv\left\langle n_{m}\right\rangle$ and $n_{f} \equiv\left\langle\nu_{i}\right\rangle$ are average occupancies. The first three terms represent the projected starting Hamiltonian with residual (projected) hybridization only. The next three represent, respectively: the Kondo interaction, the superexchange part and the interaction of Dzialoshinskii-Moriya-type, the last appearing only if the $c$-electrons are present. The noncollinearity of the magnetic ordering of $c$ electrons $\left(\sim \hat{\boldsymbol{S}}_{i} \cdot\left(\hat{\boldsymbol{s}}_{n} \times \hat{\boldsymbol{s}}_{m}\right)\right)$, as well as the superexchange interaction between them, were neglected in effective Hamiltonian (13) since the $c$ bandwidth $W_{\mathrm{c}}=2 z\left|t_{\langle m n\rangle}\right|$ is by far the largest energy in the $c$-electron subsystem.

The corresponding exchange integrals have the following forms:

$$
\begin{aligned}
& J_{i m}^{(\mathrm{K})} \equiv 2 \frac{\left|V_{i m}\right|^{2}}{U+\epsilon_{f}}-4 \frac{\left|V_{i m}\right|^{4}}{\left(U+\epsilon_{f}\right)^{3}} \\
& -4 \sum_{n(i)} \frac{\left|V_{i m}\right|^{2}\left|V_{i n}\right|^{2}}{\left(U+\epsilon_{f}\right)^{3}}\left(1-\frac{n_{c}}{2}\right) \\
& -2 \sum_{n(i)} \frac{\left|V_{i m}\right|^{2}\left|V_{i n}\right|^{2}}{\left(U+\epsilon_{f}\right)^{3}} n_{c}-2 \sum_{j(m)} \frac{\left|V_{i m}\right|^{2}\left|V_{j m}\right|^{2}}{\left(U+\epsilon_{f}\right)^{3}} n_{f}, \\
& J_{i j}^{(\mathrm{H})} \equiv \sum_{m(i)} \frac{\left|V_{j m}\right|^{2}\left|V_{i m}\right|^{2}}{\left(U+\epsilon_{f}\right)^{3}} n_{c} .
\end{aligned}
$$

The first of them represents the effective Kondo exchange integral calculated here to the fourth order; the second, the exchange integral for both the Heisenberg part and the novel three-spin interactions. Note that in order to estimate the corresponding exchange integrals, the average occupancies $n_{c}$ and $n_{f}$ have been taken for the actual occupancies. Obviously, $n_{\mathrm{e}}=n_{c}+n_{f}$. Now, we can estimate numerically the values of (14) and (15), as discussed next.

\section{Estimates of exchange integrals}

The numerical estimates of the exchange integrals appearing in (14) and (15) are shown in Figs. 3 and 4 for the two values of Coulomb interaction $U: \epsilon_{f}+U=3 \mathrm{eV}$ and $\epsilon_{f}+U=5 \mathrm{eV}$, respectively. We have also assumed that hybridization has nonzero value only for nearest neighbours $V_{\langle i m\rangle}=V$, where the number of nearest neighbors $z=4$ and the hybridization magnitude $|V|=0.3 \div 0.5 \mathrm{eV}$. Typically for Ce systems the number of electrons per site is $n_{c}=1$ and $n_{f}=1$. Let us note that to estimate $J_{i j}^{(\mathrm{H})}$ we assume that sites $i$ and $j$ are next nearest neighbors, such that summation in (15) allows only those $m$, which are nearest neighbors with both $i$ and $j$. 


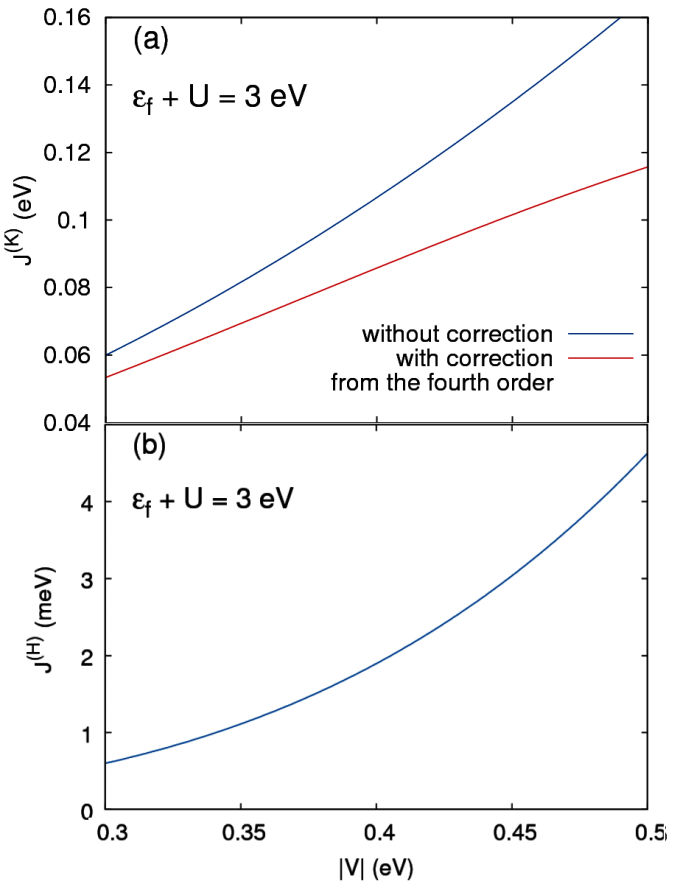

Fig. 3. Exemplary values of the Kondo exchange integral $J^{(\mathrm{K})}$ with and without correction from the fourth order (a) and that for the superexchange integral $J^{(\mathrm{H})}$ (b); both as a function of bare hybridization magnitude $|V|$, for $\epsilon_{f}+U=3 \mathrm{eV}$.

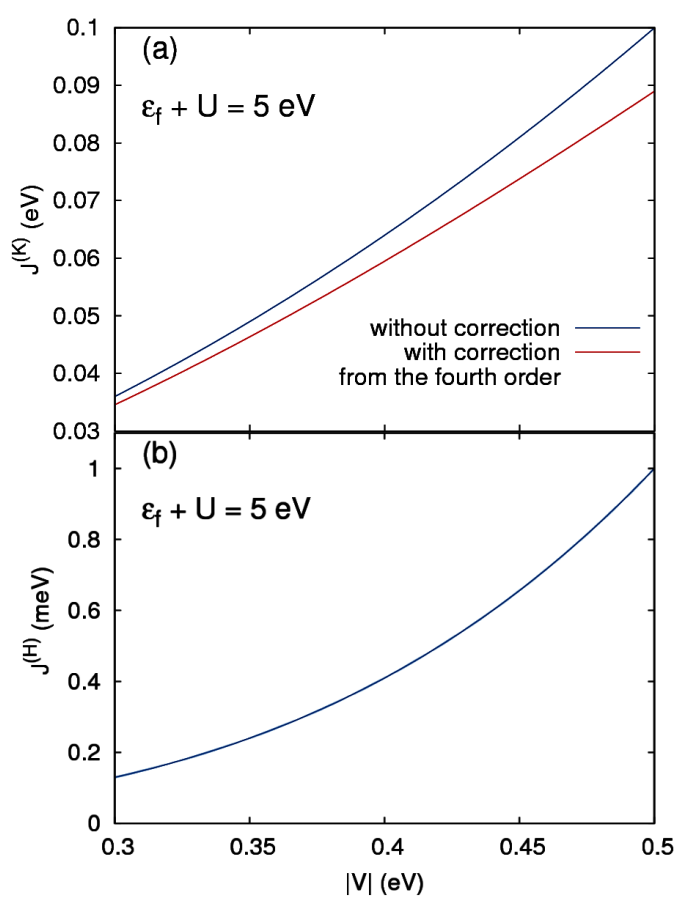

Fig. 4. Values of the Kondo exchange $J^{(\mathrm{K})}$ with and without correction coming from the fourth order (a) and of superexchange $J^{(\mathrm{H})}$ integral (b); both integrals as a function of bare hybridization magnitude $|V|$, and for $\epsilon_{f}+U=5 \mathrm{eV}$.
Let us note that $J^{(\mathrm{K})}$ in Fig. 3a is always antiferromagnetic; the fourth order effects reduce the second-order value by $\approx 30 \%$ for the smaller $U$-value. Likewise, the $f-f$ exchange $J^{(\mathrm{H})}$ is also always antiferromagnetic and more than an order of magnitude smaller, as it should be, since it contains solely the fourth-order processes. For the larger value of $U$ the integral $J^{(\mathrm{H})}$ and the correction from the fourth order in $J^{(\mathrm{K})}$ are smaller. Let us note also that the present approach contains short range interaction between asymptotically itinerant fermions $\left(V_{i m} \neq 0\right)$.

\section{Concluding remarks}

The value of the Kondo exchange and the superexchange integrals have been evaluated as a function of hybridization magnitude. In the metallic state there appears a 3-spin interaction (the last term in (13)), which may introduce a noncollinearity of the spins in the magnetic heavy-fermion state. A detailed analysis of the results will be published separately.

\section{Acknowledgments}

The work was supported in part by the project TEAM awarded to our group by the Foundation for Polish Science (FNP) for the years 2011-2014, as well as by the grant MAESTRO from the National Science Centre (NCN), No. DEC-2012/04/A/ST3/00342.

\section{References}

[1] J. Spałek, P. Gopalan, J. Phys. (France) 50, 2869 (1989).

[2] O. Howczak, J. Spałek, J. Phys. Condens. Matter 24, 205602 (2012).

[3] O. Howczak, J. Kaczmarczyk, J. Spałek, Phys. Status Solidi B 250, 609 (2013).

[4] J.R. Schrieffer, P.A. Wolff, Phys. Rev. 149, 491 (1966). 